DOI: $10.17957 / \mathrm{IJAB} / 15.1800$

http://www.fspublishers.org

\title{
New Record of Jumping Plant Lice, Trioza hirsuta (Hemiptera: Triozidae) and its Associated Parasitoid Psyllaephagus phylloplectae (Hymenoptera: Chalcidoidea: Encyrtidae) from Pakistan
}

\author{
Muhammad Tariq Rasheed ${ }^{1 \dagger}$, Imran Bodlah ${ }^{1 * \dagger}$, Muhammad Farooq Nasir ${ }^{1}$ and Tariq Mahmood $^{2}$ \\ ${ }^{1}$ Insect Biodiversity and Conservation Group, Department of Entomology, Pir Mehr Ali Shah Arid Agriculture University, \\ Rawalpindi, Pakistan \\ ${ }^{2}$ Department of Environmental Sciences, Pir Mehr Ali Shah Arid Agriculture University, Rawalpindi, Pakistan \\ *For correspondence: imranbodlah@gmail.com \\ †Contributed equally to this work and are co-first authors \\ Received 12 September 2020; Accepted 17 March 2021; Published 10 May 2021
}

\begin{abstract}
Trioza hirsuta (Crawford 1912), a potential pest of Terminalia arjuna (Roxb.) Wight and Arn 1834 along with its parasitoid, Psyllaephagus phylloplectae Sushil and Khan (1995) is hereby reported for the first time from Pothwar region of Pakistan. Detailed description and distributional detail for both the taxa is provided. In the study, trophic associations of ants with $T$. hirsuta were also studied. It was noticed that 9 ants' species are associated with this pest. Images of psyllid and its associated parasitoid are given to facilitate future identifications. Distribution map of the species has been given using Arc GIS Tools. (C) 2021 Friends Science Publishers
\end{abstract}

Keywords: Psyllid; Parasitoid; Ants; Distribution; Pakistan

\section{Introduction}

Psyllids or jumping plant-lice (Hemiptera: Psylloidea) are phytophagous, sap-sucking insect pest of various host plants (Hodkinson 2009). Economically these pests are responsible for significant economic losses and act as vector for disease causing plant pathogens (Aubert 1987). The association between plants and psyllids results in plant injuries that appear in the form of galls, leaf curling and lerp development (Burckhardt 2005). Besides, few species of psyllids are potential bio control agents of mosquitoes and weeds as well (Donnelly 2002; van Klinken et al. 2003). The genus Trioza has been reported as one of the most distributed genera of psyllid throughout the world. According to Hollis (1984), individuals of genus Trioza can be identified on the basis of following characters; vertex having median suture at anterior margin; head and mesosoma glabrous with few setae; forewing narrowsubangular apically and elongated morphologically; cells $\mathrm{m} 1, \mathrm{~m} 2$ and $\mathrm{Cu} 1$ having radular part separately; apical spur absent in basal tarsal segment of hind leg; generally unipartite proctiger in male.

Across the globe a record of 4000 species of psyllids has been documented (Li 2011). Major works on genus Trioza include studies of Crawford (1914), Hodkinson and White (1981), Hodkinson (1984), Hollis (1984), Burckhardt
(2005), Yang et al. (2006), Burckhardt (2007), Yang and Raman (2007), Hodkinson (2009), Ouvrard et al. (2015) and Burckhardt et al. (2018). Among neighboring countries to Pakistan, documented taxa under genus Trioza includes three species of from Afghanistan (Malenovský et al. 2012), eight species from Iran (Zendedel et al. 2016) and 43 species from India (Burckhardt et al. 2018). However, little is known about this genus from Pakistan (Bodlah et al. 2012; Burckhardt et al. 2018). Moreover, Psyllaephagus phylloplectae was originally described from Uttaranchal (India) by (Sushil and Khan 1995) on its host, Trioza hirsuta. However, P. phylloplectae along with its host, $T$. hirsuta has also been recorded from Indian state of Karnataka (Gupta et al. 2009). Herein we recorded it as a new faunal record from Pakistan.

\section{Materials and Methods}

During 2017-2019, several surveys were conducted for the collection of jumping plant lice along with its parasitoid and mutualistic associated ants from district Rawalpindi and Islamabad, Pakistan.

Collection and identification of psyllid: Adult psyllids were collected from Terminalia arjuna by conducted extensive surveys in Pothwar region Infested leaves were collected and placed in plastic jars which were then shifted

To cite this paper: Rasheed MT, I Bodlah, MF Nasir, T Mahmood (2021). New record of jumping plant lice, Trioza hirsuta (Hemiptera: Triozidae) and its associated parasitoid Psyllaephagus phylloplectae (Hymenoptera: Chalcidoidea: Encyrtidae) from Pakistan. Intl J Agric Biol 25:1375-1382 
to laboratory and placed at room temperature for the emergence and collection of adults and all nymphal stages. Adult psyllids were preserved in $75 \%$ ethanol. Few of the specimens were mounted on triangular card for identification purpose. For dissection of various body parts and extraction of genitalia methodology of Bodlah et al. (2012) was followed. Microscopic slides mounted with canada balsam were prepared for immature specimens; separate for each nymphal instar. All collected specimens were identified up to species level following Mathur (1975) and examining under Leica MS 5 stereomicroscope. Images of adult and immature stages were prepared through Amscope 18 megapixel camera attached with NOIF XSZ 107 BN Slide microscope. Prepared images were cleaned in Adobe Photoshop CS6 software. Measurements of different body parts were taken with the help of stage and ocular micrometer.

Collection and identification of parasitoid: During collection of adult psyllids, mummified psyllids were also collected and placed in small petri dishes. These were then shifted to laboratory and placed at ambient temperature for the emergence of parasitoids. Emerged parasitoids were collected and preserved in $75 \%$ ethanol. Few of these were mounted on small triangular card for identification purpose. Specimens were identified up to species level using Leica MS5 stereomicroscope and following taxonomic literature of Sushil and Khan (1995), Hayat (2006) and Gupta et al. (2009). Morphometry was done using stage and ocular micrometer and images were taken as stated above.

Collection and identification of Ants foraging on honeydew: During field surveys and collection of psyllids, various species of ants were observed. These ants were found in association to T. hirsuta and foraging on honeydew secreted by psyllids. These associated ants were collected and identified up to species level following (Bingham 1903). All identified specimens (psyllid, parasitoid and ants) were deposited at Laboratory of Insect Biodiversity and Conservation, Department of Entomology, Pir Mehr Ali Shah Arid Agriculture University Rawalpindi.

Following abbreviations are used in description and morphometric analysis of psyllid and its parasitoids. All measurements are in millimeter $(\mathrm{mm})$.

BL: Body length (total length of body from head to genital plate); BW: Body Width in dorsal view (maximum wider part of body excluding wing pad); HW: Head width in frontal view; AL: Antennal length (total length of antennae from basal segment to apical one); SL: Scape length; ML: Mesosomal length (length of mesosoma in profile); MW: Mesosomal width (width of mesosoma dorsally); WL: Length of fore wing; wL: Length of hind wing; MTL: Length of Metatibia; MFL: Length of meta femur; MPL: Length of male proctiger; PL: Length of paramere; DAL: Aedeagus length (distil segment length); FPL: Length of female proctiger; F1-6: Funicular segment one-six of antennae.

\section{Results}

Jumping plant lice Trioza hirsuta and its associated parasitoid Psyllaephagus phylloplectae have been recorded for the first time from Pakistan. The ecological interaction of $T$. hirsuta with ants has also been observed. The distribution pattern of both taxa in Pakistan is provided (Fig. 6).

\section{Trioza hirsuta (Crawford 1912)}

Systematic account: Kuwayama hirsuta (Crawford 1912); Kuwayama hirsuta (Crawford 1924); Kuwayama hirsuta (Mathur 1975); Phylloplecta hiruta (Mani 1948); Megatrioza hirsuta (Gupta et al. 2009).

\section{Morphometry $\mathbf{n = 5}$ (Adult)}

BL: 2.71-3.14; BW: 1.03-1.08; WL: 4.8-4.92; wL: 2.683.32; MTL: 0.89-1.03; MFL: 0.52-0.67; HW: 0.90-0.94; AL: 1.99-2.13; MPL: 0.40-0.44; PL: 0.19-0.26; DAL: 0.25-0.37; FPL: 1.04-2.01.

Description Adult: Body length 2.54-2.85 mm; integument with long, thick, yellowish-light brownish setae throughout dorsum and laterally (Fig. 3a-b); Body with minute sculpture (Fig. 2a); Head not inclined, almost equal to longitudinal body axis (Fig. 2a); wider than pronotum and mesoscutum; vertex trapezoidal, dorsum prominently pointed posteriorly, about $1 / 2$ time as broad as long, in the middle of each half sulcately impressed, indented with two foveae (Fig. 2a), with long, abundant setae, apically curved towards antennal margin dorsally (Fig. 2a), occipital margin processed to inveginated, occipital regions strongly produced upward, genal process absent (Fig. 2a), covered with basal segment of antennae. Eyes hemispherical, large, well defined. Clypeus oval shaped, bearing four pair of long unequal setae, clearly visible in frontal view (Fig. 2a), labrum somewhat smaller. Antennae slender, 10 segmented (Fig. 2b), 0.24-0.26 times as long as head width, basal two segments robust transversally (Fig. 2b), segment III and IV almost equal, segment V and VII nearly equal, segment VI somewhat longer than V, segment VIII shorter than segment VII, terminal segment having two short spines apically, unequal in length, segment IV, VI, VIII and IX having single rhinarium. Pronotum arched, narrower, anterior margin arcuate dorsally, about $1.5 \mathrm{X}$ as broad as long, relatively flat; mesopraescutum somewhat longer than wide dorsally, slightly arched and narrower laterally; mesoscutum relatively flat, massive, about $2 \mathrm{X}$ as broad as long dorsally, angular at anterior and posterior margins; forewing weakly lanceolate (Fig. 2c), hyaline, about $2.5 \mathrm{X}$ as long as broad, broader medially; veins beset with fine microscopic setae in high magnification; veins $\mathrm{R}, \mathrm{M}$ and $\mathrm{Cu}$ originating from same point; vein $R_{1}$ equal in length to $\mathrm{Cu}$; vein $\mathrm{R}$ somewhat longer than $\mathrm{Cu}$; vein $\mathrm{M}$ ending at apex of wing; hind wing shorter comparatively; veins beset with short, simple, hooked like microscopic setae (Fig. 2d). 
(a)

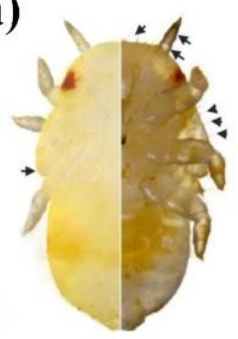

(c)

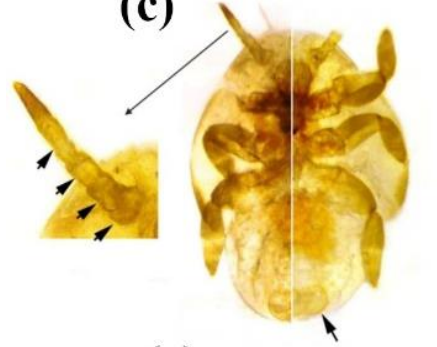

(b)

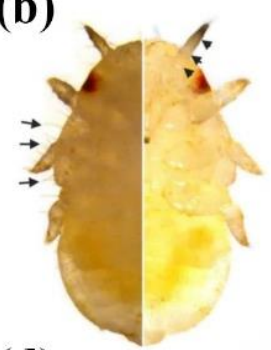

(d)

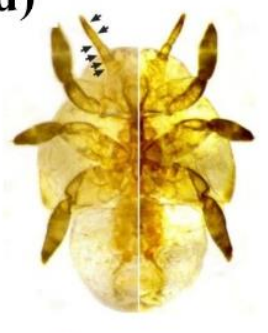

(e)

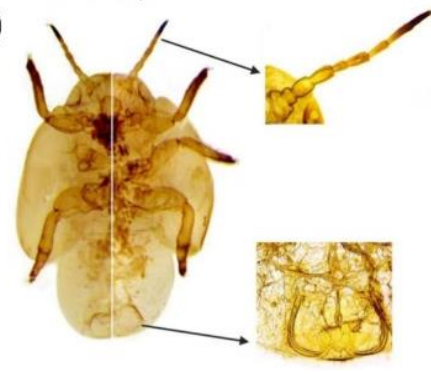

Fig. 1 (a-e): Immatures: Trioza hirsuta (Crawford 1912) (a) First instar (b) Second instar (c) Third instar (d) Fourth instar (e) Fifth instar

Abdomen relatively shorter than thorax, beset with fine pubescent sparsely (Fig. 3a, b), bearing short, arranged line points. Legs longer, pubescent, having minute points; tibiae longer than femora (Fig. 3a, b); 6-8 long, blunt setae present near apical junction of hind femur; meracanthus triangular.

Male terminalia: Male proctiger long, covered with unequal, thick setae across one third of apical region, broadest in middle (Fig. 2e); sub genital plate globular (Fig. 2e), beset with moderately long and sparsed setae, distance between setae unequal; paramere stout, with short conspicuous setae at posterior half (Fig. 2e), slightly dens apically; aedeagus longer, terminal face shorter than basal (Fig. 2e), slightly curved medio apically (Fig. 2e).

Female terminalia: Female proctiger $1.5 \mathrm{X}$ as long as broad, bearing short, minute setae medially (Fig. 2f), long, unequal setae present at apical region; sub genital plate $0.5 \mathrm{X}$ as broader as long, bearing dense, unequal setae across one fourth; lateral valve rounded (Fig. 2e), bearing abundant of short setae in profile (Fig. 2e).

Fifth instar: Body $2.37-3.24 \mathrm{~mm}$ in length, body width $1.03-1.25 \mathrm{~mm}$ dorsally; Forewing pad produced posteriorly, about 1.44-1.63 mm long on dorsum; whole body sclerotic, whilst median line membranous throughout, six pairs of
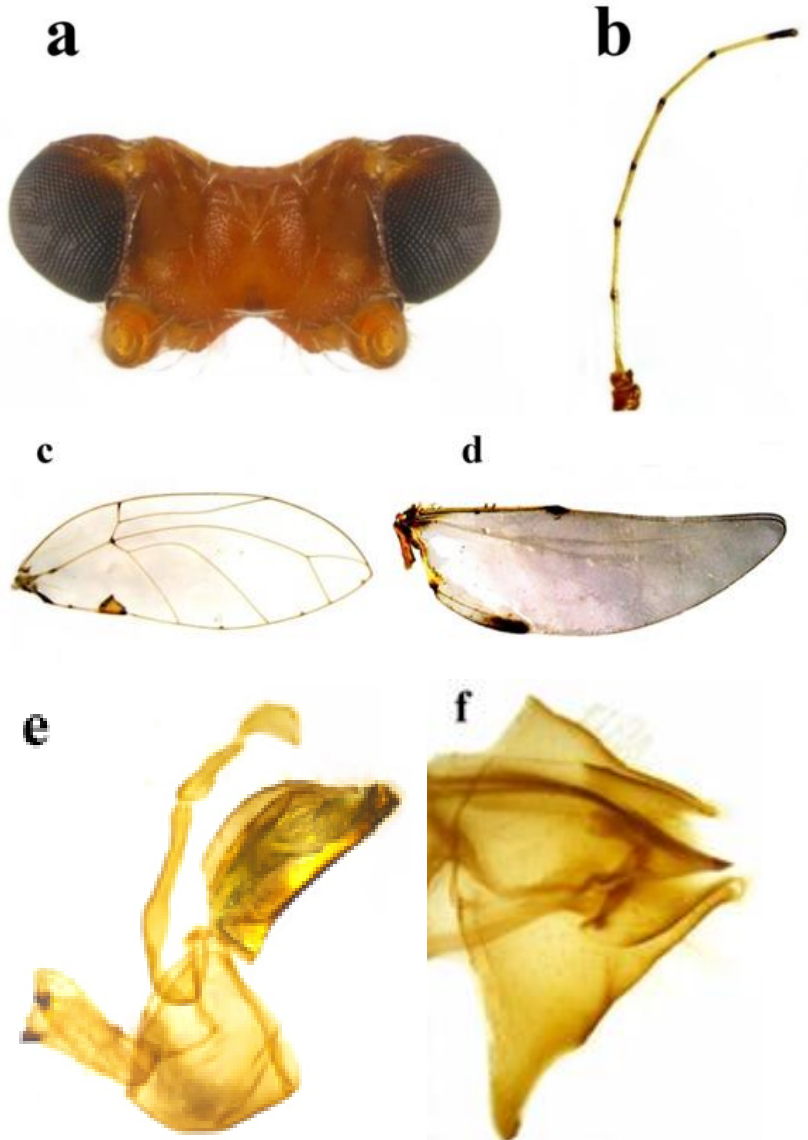

d
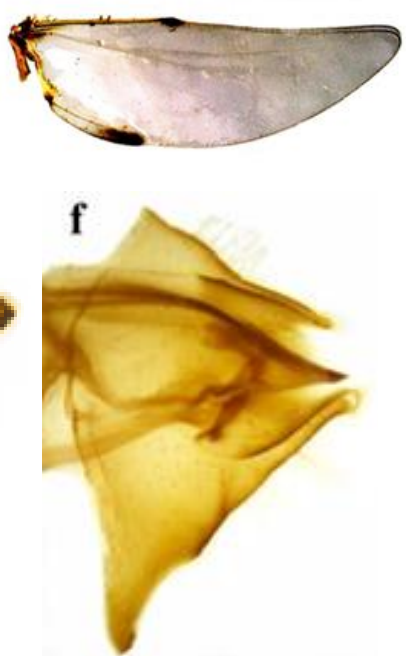

Fig. 2: Trioza hirsuta (Crawford, 1912) (a-f) (a) Head (b) Antennae (c) Forewing (d) Hind wing (e) Male terminalia (f) Female terminalia

sclerotic strips present over abdomen; head, mesosoma and metasoma bearing poorly vermiculated derm along with microscopic points and simple ring-based setae of unequal length; antennae eight segmented (Fig. 1e), short and slender, total antennal length $0.76-0.89 \mathrm{~mm}$ with first segment $0.1-0.26 \mathrm{~mm}$, second segment $0.04-0.10 \mathrm{~mm}$, third segment $0.10-0.11 \mathrm{~mm}$, fourth segment $0.12-0.21 \mathrm{~mm}$, segment five $0.06-0.07 \mathrm{~mm}$, segment six $0.11-0.12 \mathrm{~mm}$, segment seven $0.10-0.11 \mathrm{~mm}$ and segment eight $0.25-0.27$ $\mathrm{mm}$ long (bearing two short spines of variable length; vertex length $0.38-0.50 \mathrm{~mm}$ dorsally; Legs short, beset with simple setae; ring pores at abdominal end (Fig. 1e).

Fourth instar: Body1.64-1.67 mm longer in dorsal view; body width $0.58-0.72 \mathrm{~mm}$ dorsally. Triozine form, similar to fifth instar; antennae six segmented (Fig. 1d), total antennal length $0.43-0.48 \mathrm{~mm}$, basal segment $0.05-0.07$ mm longer, segment second $0.04-0.05 \mathrm{~mm}$, segment third 0.04-0.06 mm, segment four $0.08-0.10 \mathrm{~mm}$, segment five $0.05-0.06 \mathrm{~mm}$, segment six $0.16-0.18 \mathrm{~mm}$; vertex length $0.52-0.56 \mathrm{~mm}$ in dorsal view.

Third instar: Body $0.95-1.28 \mathrm{~mm}$ longer in dorso flattened view; body width $0.43-0.58 \mathrm{~mm}$. Forewing pad smaller 

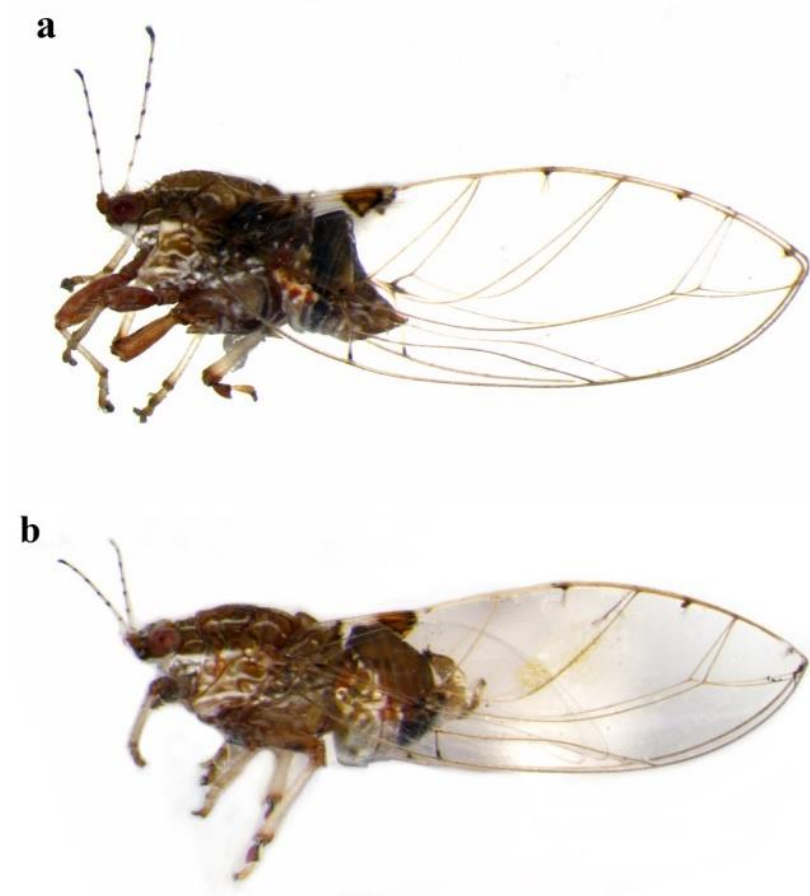

Fig. 3: Adult, Trioza hirsuta (Crawford 1912) (a-b): (a) Female; lateral view (b) Male; lateral view

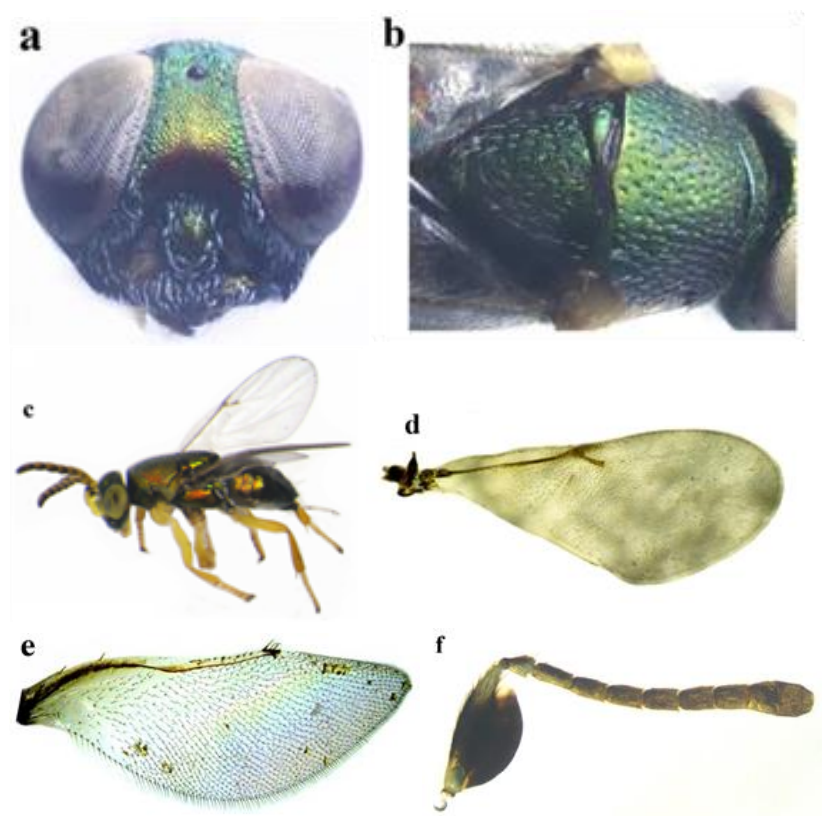

Fig. 4: Adult (Female), Psyllaephagus phylloplectae (a-f) (a) Head; frontal view (b) Thorax; dorsal view (c) Body; lateral view (d) Fore wing (e) Hind wing (f) Antennae

than fourth instar. Antennae four segmented (Fig. 1c), total antennal length $0.032-0.035 \mathrm{~mm}$, basal segment $0.03-0.04$ $\mathrm{mm}$ in length, segment second $0.04-0.05 \mathrm{~mm}$, segment third $0.07-0.09$, segment fourth $0.16-0.19 \mathrm{~mm}$ longer. Head with three pair of setae. Legs very shorter than fourth instar.
Portion of ring pore clearly visible in dorso-flattened view (Fig. 1c).

Second instar: Body $0.50-1.0 \mathrm{~mm}$ in length, body width $0.51-0.56 \mathrm{~mm}$. Forewing pad much smaller and knob like. Antennae three segmented (Fig. 1b), total antennal length $0.11-0.13 \mathrm{~mm}$, segment first $0.04-0.05 \mathrm{~mm}$ longer, segment second 0.04-0.05 mm, segment three 0.05-0.06 mm. Abdomen beset with lanceolate setae (Fig. 1b).

First instar: In dorsal view, body $0.30-0.76 \mathrm{~mm}$. Antennae with two segments (Fig. 1a), total antennal length $0.12-0.13$ $\mathrm{mm}$, segment first $0.03-0.04 \mathrm{~mm}$, segment second 0.09 $0.10 \mathrm{~mm}$ in length.

Material examined: Islamabad: Daman-e-koh

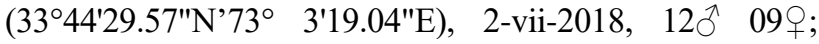
Rawalpindi: PMAS Arid Agriculture University

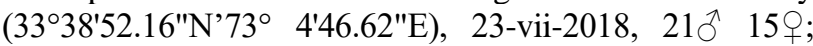
Kahuta $\left(33^{\circ} 35^{\prime} 20.90^{\prime \prime} N^{\prime} 73^{\circ} 23^{\prime} 49.39^{\prime \prime E}\right), 28$-vii-2018, $17{ }^{\lambda}$ 11 ; ; Islamabad: Sihala $\left(33^{\circ} 33^{\prime} 7.99^{\prime \prime} \mathrm{N}^{\prime} 73^{\circ} 12^{\prime} 18.62^{\prime \prime E}\right), 28-$ vii-2018, $07 \mathrm{O}^{\circ} \quad 10$; $\quad$ Rawalpindi

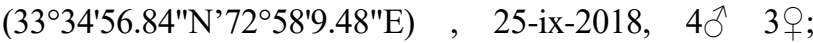
Islamabad : Rose and Jasmine Garden

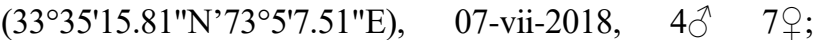
Islamabad : Faisal Mosque (3343'37.77"N'73²'18.44"E), 12-vii-2018, $12 \bigcirc^{\Uparrow} 09 \bigcirc$; Rawalpindi : Morgah Biodiversity

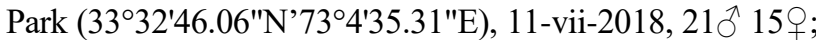

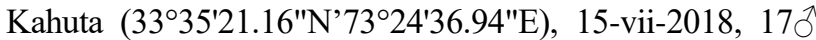
11 ; Islamabad : Pakistan Agricultural Research Council (334' 12.96"N'735'43.51"E), 17-vii-2018, 07ð 10ㅇ; Islamabad : Village Malpur (3343'30.94"N'739'6.25"E),

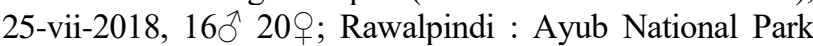
(3334'19.15"N'734'59.05"E), 5-viii-2018, $16 \overbrace{}^{\wedge} 20$ \% Rawalpindi (333 $\left.33^{\prime} 55.56^{\prime \prime} \mathrm{N}^{\prime} 73^{\circ} 0^{\prime} 57.03^{\prime \prime E}\right), \quad 13$-viii-2018, $10{ }^{\wedge}$ 07웅 Rawalpindi : Morgah Biodiversity Park

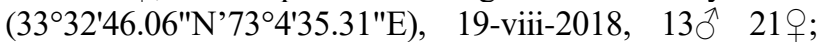
Rawalpindi : Nawaz Sharif Park (3338'57.86"N'734'32.64"E), 26-viii-2018, 4خ 9; Rawalpindi : PMAS Arid Agriculture University

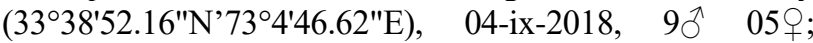
Islamabad : Shahdara $\quad$ Valley

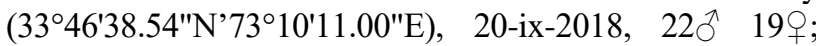
Islamabad : F-9 Park (3342'6.47"N'73²1'24.96"E), 25-ix2018, 40 3ㅇ Islamabad : Kachnar Park

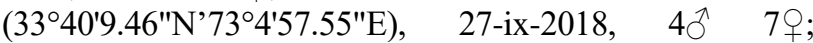

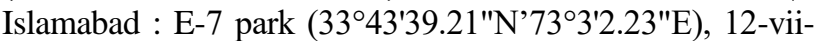
2019, $12 \bigcirc^{\Uparrow} 09$; Rawalpindi : Morgah Biodiversity Park $\left(33^{\circ} 32^{\prime} 46.06^{\prime \prime}{ }^{\prime} 73^{\circ} 4^{\prime} 35.31^{\prime \prime E}\right), 21-v i i-2018,21 \delta^{\lambda} 15$ 우 Kahuta $\left(33^{\circ} 35^{\prime} 21.16^{\prime \prime} \mathrm{N}^{\prime} 73^{\circ} 24^{\prime} 36.94 " \mathrm{E}\right), 22$-vii-2019, $17 ð$ 119; Islamabad : Lake view park

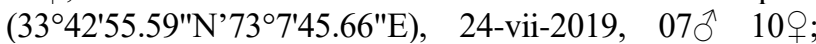
Islamabad : Village Malpur (3343'30.94"N'739'6.25"E), 25-viii-2019, $16 \bigcirc^{\lambda} \quad 209$; Rawalpindi (3338'57.86"N'734'32.64"E), 09-viii-2019, $10 \overbrace{}^{\top} 07$;

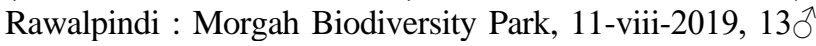
21 ; Rawalpindi (3338'52.16"N'734'46.62"E), 13-viii-19, 31 $0^{\lambda}$ 22ᄋ; Rawalpindi : Nawaz Sharif Park

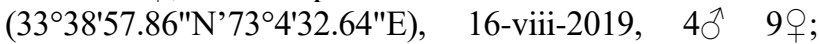


Rawalpindi :PMAS Arid Agriculture University $\left(33^{\circ} 38^{\prime} 52.16^{\prime \prime} \mathrm{N}^{\prime} 73^{\circ} 4^{\prime} 46.62^{\prime \prime} \mathrm{E}\right), \quad 04-\mathrm{ix}-2019, \quad 9 \overbrace{}^{\prime} \quad 05$ 웅

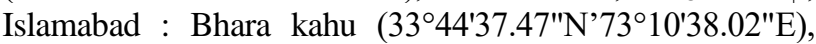
02-ix-2019, 220 19๑; Islamabad : F-9 Park

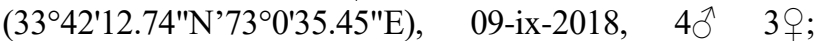

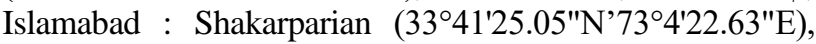
13-ix-2019, 4ð 7웅 Rawalpindi, 09-viii-2019, 10^ 07우; Rawalpindi : Morgah Biodiversity Park

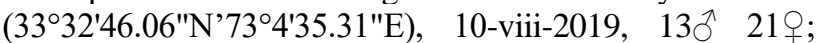
Rawalpindi : Chattar park (3346 $\left.43.35^{\prime \prime} \mathrm{N}^{\prime} 73^{\circ} 14^{\prime} 30.48^{\prime \prime} \mathrm{E}\right)$, 15-viii-2019, $4 \bigcirc^{\wedge} 9 \circ$; Islamabad : Sohan

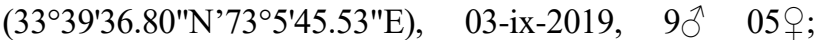

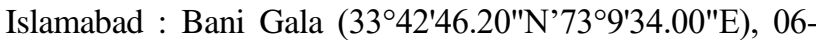
ix-2019, 22 $\bigcirc^{\wedge} 19$; Islamabad : National Agricultural Research Centre (3340'1.92"N'737'22.32"E), 01-ix-2019, $40^{\lambda} \quad 3 \circ$; Islamabad : $\mathrm{H}-9$ Sector (3340'20.87"N'733'18.05"E) 11-ix-2019, 4ð 7 우.

Global Distribution: India, Sri Lanka (Burckhardt et al. 2018); Pakistan (this study).

Host plant: Individuals were collected from marginal curved leaves of Terminalia arjuna.

Field observations: Trioza hirsuta was observed as a serious pest of $T$. arjuna at various localities of Rawalpindi and Islamabad. High infestation of this pest leads to folding and curling of marginal parts of leaf blades (Fig. 5a, b). Immature remain inside the folded leaves (Fig. 5c), whilst adults were observed on leaves and tiny branches of host plant for continuous feeding of plant sap (Fig. 5d).

Ants foraging over honeydew: Islamabad (Daman-e-koh), 2-vii-2018, 12ఫ (Camponotus compressus, Tapinoma melanocephalum, Lepisiota frauenfeldi); Rawalpindi : PMAS Arid Agriculture University, 23-vii-2018, 21ષ (Meranoplus bicolor, Tapinoma melanocephalum, Camponotus compressus, Lepisiota frauenfeldi, Lepisiota opaca pulchella, Monomorium sagei); Kahuta, 28-vii-2018, 17ð̧ (Lepisiota capensis simplex, Tapinoma melanocephalum, Monomorium sagei); Islamabad : Sihala, 28-vii-2018, 07ðॄ (Camponotus compressus, Lepisiota opaca pulchella, Crematogaster subnuda); Rawalpindi , 25ix-2018, 4ఫ్ (Camponotus compressus, Lepisiota capensis simplex, Tapinoma melanocephalum, Lepisiota opaca pulchella, Lepisiota frauenfeldi); Islamabad (Rose and Jasmine Garden), 07-vii-2018, 7ఫ (Lepisiota capensis simplex, Tapinoma melanocephalum, Monomorium sagei, Crematogaster subnuda); Islamabad (Faisal Mosque), 12vii-2018, 12ช్; Rawalpindi (Morgah Biodiversity Park), 11vii-2018, 21ర(Tapinoma melanocephalum, Meranoplus bicolor, Monomorium sagei); Kahuta, 15-vii-2018, 17ర్(Plagiolepis jerdonii, Tapinoma melanocephalum); Islamabad : Pakistan Agricultural Research Council, 17-vii2018, 07ð̧ (Tapinoma melanocephalum, Meranoplus bicolor, Camponotus compressus); Islamabad : Village Malpur, 25-vii-2018, 16ర్(Camponotus compressus,
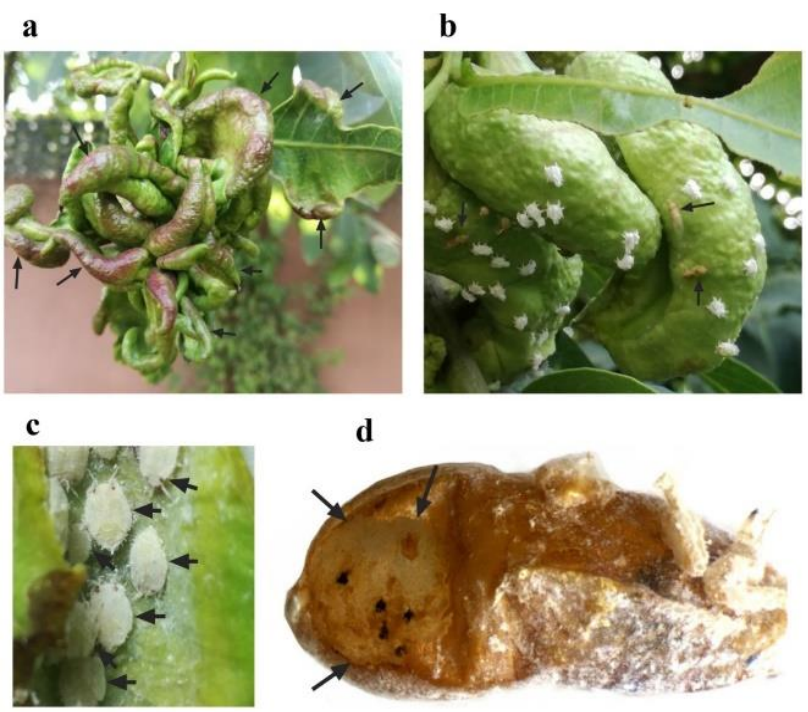

Fig. 5: (a-d): (a-b) Marginal leaf galls of Terminalia arjuna (c) Psyllid infestation (immatures) (d) Irregular hole pattern after emergence of parasitoid from fifth instar

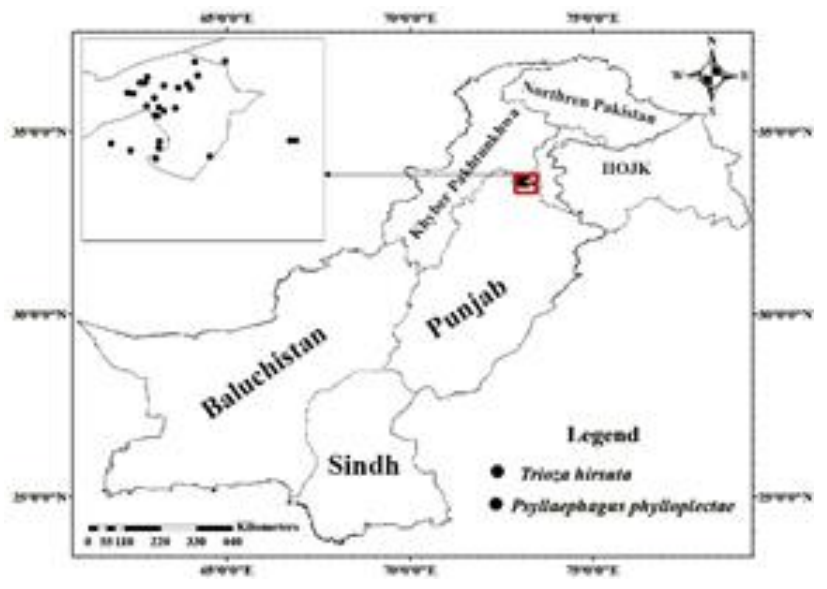

Fig. 6: Distribution pattern of T. hirsuta and P. phylloplectae in Pakistan

Plagiolepis jerdonii); Rawalpindi : Ayub National Park, 5viii-2019, 16ఫ (Tapinoma melanocephalum, Meranoplus bicolor, Camponotus compressus); Rawalpindi, 13-viii2019, 10ళ (Camponotus compressus, Tapinoma melanocephalum, Lepisiota frauenfeldi); Rawalpindi : Morgah Biodiversity Park, 19-viii-2019, 13ఫ్ (Camponotus compressus, Tapinoma melanocephalum); Rawalpindi, 22viii-2019, 31రॄ (Monomorium sagei, Camponotus compressus, Meranoplus bicolor); Rawalpindi : Nawaz Sharif Park, 26-viii-2019, 4ర్ (Lepisiota capensis simplex, Tapinoma melanocephalum, Monomorium sagei, Crematogaster subnuda); Rawalpindi : PMAS Arid Agriculture University, 04-ix-2019, 9ð (Tapinoma melanocephalum, Meranoplus bicolor, Camponotus 
compressus, Lepisiota frauenfeldi, Lepisiota capensis simplex, Lepisiota opaca pulchella); Islamabad: Shahdara Valley, 20-ix-2019, 22ช్ (Tapinoma melanocephalum, Monomorium sagei, Crematogaster subnuda); Islamabad: F-9 Park, 25-ix-2019, 4ช్;; Islamabad : Kachnar Park, 27-ix2019, 4ఫ్(Plagiolepis jerdonii, Tapinoma melanocephalum, Meranoplus bicolor, Camponotus compressus).

\section{Psyllaephagus phylloplectae (Sushil and Khan 1995)}

Description: Female. Length 2.48-2.56 mm (n=5).

Colour/Sculpture: Head brilliant metallic green (Fig. 4a); frontovertex metallic green with golden tinge suffusion; lower part of face reddish; ocular area metallic green with regular punctations throughout of median ocellus. Lower part of face, dorso-lateral part of scrobes, lower level of eyes, upper and lower margin of supraclypeal area, malar sulcus and clypeus with silvery whitish setae (Fig. 4a). The area from frontovertex-median ocellus bearing small-large punctations; parascrobal area greenish with large punctations. Eyes greyish, bare, ocelli dark or nearly black. Antennae pale yellow and infuscate (Fig. 4f), lacking brown setae; scape dark-nearly black, pedicel pale yellow-brown with small brown setae; funicle and clava light brown-dark brown, F1-F3 pale yellow band along ventral margin. Mandibles dark grey, maxillary and labial palpi light yellow.

Mesosoma metallic green and shining (Fig. 4b); dorsum of pro and mesonotum reticulate-punctate, the punctations relatively larger along with silvery whitish setae, the setae gradually becoming larger toward the apex of scutellum (Fig. 4b); mesoscutum relatively less number of punctations; tegula yellow-light brown with brown setae (Fig. 4b); prepectus light yellow-somewhat brown; mesopleuron infuscate and slightly golden reflection, sculptured with reticulations (Fig. 4c). Metanotum dark brown to nearly black; Legs pale yellow, claws dark brown to black. Fore wing hyaline, venations light brown (Fig. 4d), margins with pale reflections; Hind wing whitish and setigerous, venations brown-dark brown, margins pale yellow (Fig. 4e). Metasoma greenish at basal half, apical half black laterally.

Structure: Head in frontal view rounded-oval (Fig. 4a), anterior half wider, about $0.82-0.90 \mathrm{~mm}$ in width and $0.67-$ $0.71 \mathrm{~mm}$ in length; eyes strongly diverging along median ocellus, slightly straight at the level of frontovertex, gradually divergent from parascrobes-scrobes, scrobes deep and elongated with rounded torulus; torulus slightly below the lower margin of eyes; distance between torulus and mouth almost equal; distance between torulus and lower margin of eyes slightly lesser than distance between toruli; distance between torulus and median ocellus is greater than head width. Head concave in frontal view; frontovertex narrow toward median ocellus and gradually toward parascrobal area; antennae 1.12-1.60 mm in length; scape expanded (Fig. 4f), about $0.26-0.79 \mathrm{~mm}$ in length; pedicle nearly triangular; F1 slightly larger than F2-6; clava broad medially and narrow apically (Fig. 4f); F6 slightly wider than F1.

Mesosoma as long as wide, slightly wider than head; about $0.98-1.32 \mathrm{~mm}$ in length and $0.69-1.20 \mathrm{~mm}$ in width; fore wing hyaline, post marginal vein somewhat shorter than stigmal vein (Fig. 4d), setigerous about 1/4 toward apical margin; hind wing whitish, membrane with minute setae based on prominent projection. Metasoma elongated-triangular; apex narrowly pointed, ovipositor exerted (Fig. 4c).

\section{Morphometric n=5 (Adult)}

HL: 0.67-0.7; HW: 0.82-0.90; SL: 0.26-0.79; AL: 1.121.60; EL: 0.44-049; EW: 0.26-0.29; BL: 2.79-3.09; ML: 0.98-1.32; MW: 0.69-1.20; WL: 1.65-1.80.

Material examined: Islamabad: Daman-e-koh (334'29.57"N'73³'19.04"E), 2-vii-2018, $12 \overbrace{}^{\Uparrow} \quad 09$ \%; Rawalpindi: PMAS Arid Agriculture University

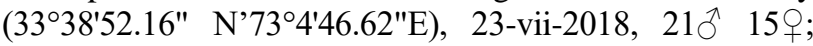
Kahuta $\left(33^{\circ} 35^{\prime} 20.90^{\prime \prime} N^{\prime} 73^{\circ} 23^{\prime} 49.39^{\prime \prime E}\right), 28$-vii-2018, $17{ }^{\jmath}$ 11 \%; Islamabad: Sihala $\left(33^{\circ} 33^{\prime} 7.99^{\prime \prime}{ }^{\prime} 73^{\circ} 12^{\prime} 18.62^{\prime \prime E}\right), 28$

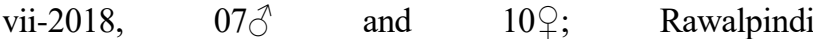

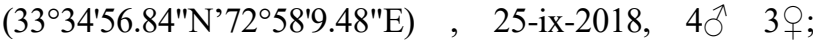
Islamabad : Rose and Jasmine Garden

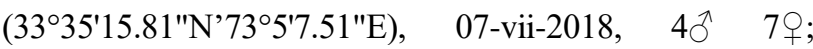

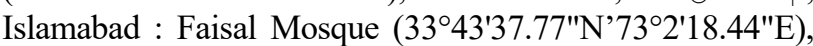

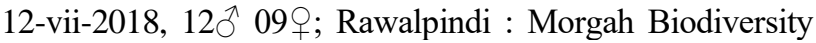
Park (3332'46.06"N'734'35.31"E), 11-vii-2018, $21 \overbrace{}^{\Uparrow} 15$ 웅 Kahuta $\left(33^{\circ} 35^{\prime} 21.16^{\prime \prime}{ }^{\prime} 73^{\circ} 24^{\prime} 36.94 " E\right), 15$-vii-2018, $17 \jmath$ 11 ; ;slamabad : Pakistan Agricultural Research Council

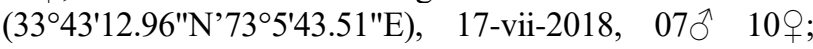
Islamabad : Village Malpur (3343'30.94"N'739'6.25"E), 25-vii-2018, $16 \bigcirc^{\top}$ 20; Rawalpindi : Ayub National Park (3334'19.15"N'734'59.05"E), 5-viii-2018, $16 \overbrace{}^{\wedge}$ 20웅 Rawalpindi (333'⒌56"N'730'57.03"E), 13-viii-2018, $10{ }^{\wedge}$ 07우 Rawalpindi : Morgah Biodiversity Park

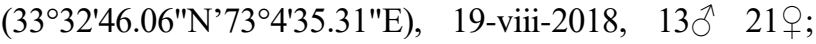
Rawalpindi: Nawaz Sharif $\quad$ Park (3338'57.86"N'734'32.64"E), 26-viii-2018, 4ત 9; Rawalpindi (PMAS Arid Agriculture University

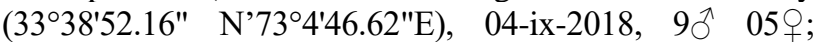
Islamabad: Shahdara Valley

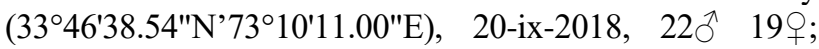
Islamabad : F-9 Park (3342'6.47"N'73²1'24.96"E), 25-ix-

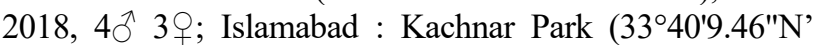
734'57.55"E), 27-ix-2018, 4๐ 7웅 Islamabad : E-7 park

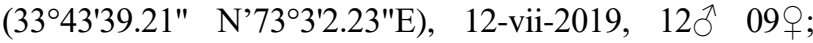
Rawalpindi: Morgah Biodiversity Park

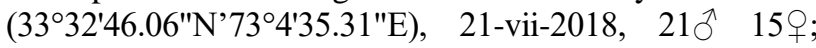
Kahuta $\left(33^{\circ} 35^{\prime} 21.16^{\prime \prime} N^{\prime} 73^{\circ} 24^{\prime} 36.94^{\prime \prime E}\right), 22$-vii-2019, $17{ }^{\jmath}$

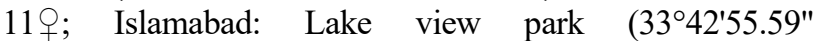
N'737'45.66"E), 24-vii-2019, 07ठ 10; Islamabad : Village Malpur (3343'30.94"N'739'6.25"E), 25-viii-2019,

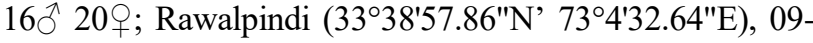


viii-2019, 10ત 07; Rawalpindi: Morgah Biodiversity

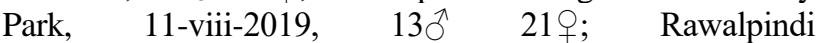

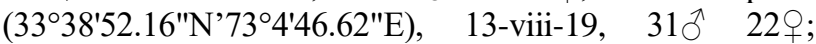
Rawalpindi :Nawaz Sharif $\quad$ Park

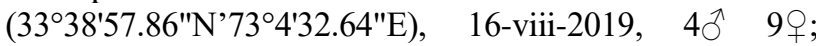
Rawalpindi (PMAS Arid Agriculture University)

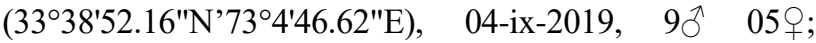

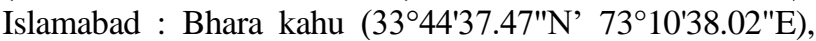
02-ix-2019, 22त 19ᄋ; Islamabad : F-9 Park

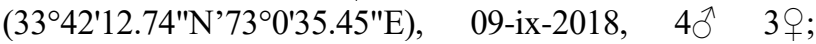

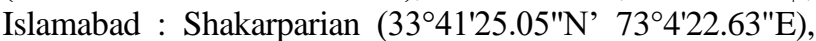
13-ix-2019, 4^ 7웅 Rawalpindi : Morgah Biodiversity Park

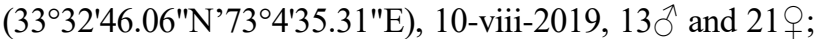
Rawalpindi : Chattar park (3346 $\left.43.35^{\prime \prime} \mathrm{N}^{\prime} 73^{\circ} 14^{\prime} 30.48^{\prime \prime} \mathrm{E}\right)$, 15-viii-2019, $4 \bigcirc^{\wedge} 99_{+}$; Islamabad : Sohan

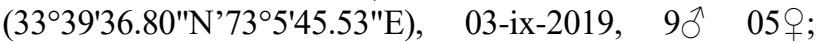

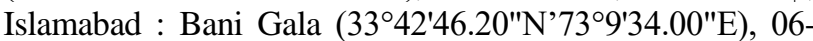
ix-2019, 22 $\hat{\sigma} 19 \%$; Islamabad : National Agricultural Research Centre (3340'1.92"N'737'22.32"E), 01-ix-2019, $4 \bigcirc^{\lambda} \quad 3$; $\quad$ Islamabad : $\mathrm{H}-9$ Sector (3340'20.87"N'733'18.05"E) 11-ix-2019, 4ð 7우.

Host: Nymph of Trioza hirsuta (Crawford 1912).

Trophic status: Parasitoid

Global distribution: India (Sushil and Khan 1995; Hayat 2006; Gupta et al. 2009); Pakistan (this study).

\section{Discussion}

Trioza hirsuta (Crawford 1912) has been observed as a potential pest of Terminalia arjuna in various localities of district Rawalpindi and Islamabad. Marginal area of leaf blade folded due to high infestation of this pest. Immatures of T. hirsuta found to live inside folded leaves, which were filled with abundant of honey dew and whitish powdery secreted material. During collection of psyllids, various species of ants were also observed viz., Camponotus compressus, Tapinoma melanocephalum, Lepisiota frauenfeldi, Lepisiota opaca pulchella, Monomorium sagei, Meranoplus bicolor, Lepisiota capensis simplex, Crematogaster subnuda and Plagiolepis jerdonii, while they were foraging over honey dew. During present work mummified psyllids were also collected. Emerged parasitoid was found similar to published description by Sushil and Khan (1995), Hayat (2006) and Gupta et al. (2009). Here Trioza hirsuta (Crawford), inducing leaf marginal galls on Terminalia arjuna (Roxb.) Wight and Arn (1834) is recorded for the first time from Pakistan along with its parasite, Psyllaephagus phylloplectae Sushil and Khan. Description of its immature stages and distribution of this psyllid in Pakistan are provided. Moreover, Psyllaephagus phylloplectae is originally described from Uttaranchal (India) by Sushil and Khan in 1995 on its host, Trioza hirsuta. However, P. phylloplectae along with its host, T. hirsuta is also recorded from Indian state of Karnataka (Gupta et al. 2009). Herein we recorded it as a new faunal record from Pakistan.

\section{Conclusion}

Trioza hirsuta (Crawford 1912) is becoming a major pest of economically important tree Terminalia arjuna (Roxb.) in various parts of district Rawalpindi and Islamabad. The damaging symptoms of $T$. hirsuta start to appear from late summer-early winter with marginal leaf galls or abnormal development of leaves. A total of nine species of ants were also found in mutualistic relationship with $T$. hirsuta while foraging over honey dew. Moreover, Psyllaephagus phylloplectae (Sushil and Khan 1995), a potential parasitoid of $T$. hirsuta was also observed, while parasitizing the nymphal stages of $T$. hirsuta. A detailed description of both newly recorded taxa along with their distribution pattern in district Rawalpindi and Islamabad are provided.

Recommendations: Further studies are needed for possible biorational pest management by mass rearing of its parasitoid and also associated natural enemies in Pakistan.

\section{Acknowledgements}

We would like to express our gratitude to Pakistan Agricultural Research Council for financial assistance under ALP funded project (CS-111) for the completion of present work. We are also thankful to Prof. Muhammad Hayat, Department of Zoology, Aligarh Muslim University, Aligarh, India, for providing the needful material for this study.

\section{Author Contributions}

IB planned the research work; MTR identified the species and wrote manuscript, MFN and TM prepared the images.

\section{Conflicts of Interest}

The authors declare that they have no conflicts of interest

\section{Data Availability}

The data used in this project available from the corresponding author on reasonable request.

\section{Ethics Approval}

Not Applicable

\section{References}

Aubert B (1987). Trioza erytreae Del Guercio and Diaphorina citri Kuwayama (Homoptera: Psylloidea), the two vectors of citrus greening disease: Biological aspects and possible control strategies. Fruits 42:149-162

Bingham CT (1903). The fauna of British India, including Ceylon and Burma. In: Hymenoptera, Vol. II, p:506. Ants and Cuckoo-wasps. Taylor and Francis, London, UK 
Bodlah I, MA Bodlah, M Naeem, T Akhter, MF Nasir, MT Chaudhry (2012). First record, distribution and morphology of Psyllid, Trioza fletcheri minor Crawford, 1912 from Punjab Province of Pakistan. Pak J Zool 44:136-1365

Burckhardt D (2007). Fauna Europaea: Psylloidea. Fauna Europaea version 1.3., Taxon Details. Available from http://www.fauna. eur.org [Accessed 7 June, 2020]

Burckhardt D (2005). Ehrendorferiana, a new genus of Neotropical jumping plant lice (Insecta: Hemiptera: Psylloidea) associated with conifers (Cupressaceae). Org Divers Evol 5:317-319

Burckhardt D, A Sharma, A Raman (2018). Checklist and comments on the jumping plant-lice (Hemiptera: Psylloidea) from the Indian Subcontinent. Zootaxa 4457:1-38

Crawford DL (1914). A monograph of the jumping plant-lice or Psyllidae of the New World. Bull US Natl Museum 85:1-182

Crawford DL (1912). Indian Psyllidae. Rec Ind Museum 7:419-435

Donnelly GP (2002). The host range and biology of the mesquite psyllid Heteropsylla texana. BioControl 47:363-371

Gupta A, V Naveenkumar, J Poorani (2009). New record of Psyllaephagus phylloplectae Sushil \& Khan (Hymenoptera: Chalcidoidea: Encyrtidae) from Karnataka, with notes on its taxonomy and host, Megatrioza hirsuta (Crawford) (Hemiptera: Triozidae). J Threat Taxa 1:174-176

Hayat M (2006). Indian Encyrtidae (Hymenoptera: Chalcidoidea), p:496. Department of Zoology, Aligarh Muslim University, Aligarh, India

Hodkinson ID (2009). Life cycle variation and adaptation in jumping plant lice (Insecta: Hemiptera: Psylloidea): A global synthesis. J Nat Hist 43:65-179

Hodkinson ID (1984). The biology and ecology of the gall-forming Psylloidea. In: The Biology of Gall Forming Insects, pp:59-77. Ananthakrishnan R (Ed.). Edward Arnold Press, London

Hodkinson ID, IM White (1981). The Neotropical Psylloidea (Homoptera: Insecta): an annotated check list. $J$ Nat Hist $15: 491-523$
Hollis D (1984). Afrotropical jumping plant-lice of the family Triozidae (Homoptera: Psylloidea). Bull Braz, Mus Nat Hist Entomol 49:1-103

Li F (2011). Psyllidomorpha of China (Insecta: Hemiptera), p:1976. Science Press, Beijing, China

Malenovský I, P Lauterer, E Labina, D Burckhardt (2012). Jumping plantlice (Hemiptera: Psylloidea) of Afghanistan. Acta Entomol Mus Nat Prag 52:1-22

Mani MS (1948). Cecidozoa and Zoocecidia from India. J Asiat Soc Beng 14:27-195

Mathur RN (1975). Psyllidae of the Indian Sub-continent, p:429. The Indian Council of Agricultural Research, New Delhi, India

Ouvrard D, D Burckhardt, C Cocquempot (2015). An annotated checklist of the jumping plant-lice (Insecta: Hemiptera: Psylloidea) from the Mercantour National Park, with seven new records for France and one new synonymy. Zoosystema 37:251-271

Sushil SN, MA Khan (1995). Two new species of Psyllaephagus (Hymenoptera: Encyrtidae) from northern India. J Ins Sci 8:20-23

van Klinken RD, G Fichera, H Cordo (2003). Targeting biological control across diverse landscapes: The release, establishment, and early success of two insects on mesquite (Prosopis spp.) insects in Australian rangelands. Biol Cont 26:8-20

Wight R, GAW Arnott (1834). Prodromus Florae Peninsulae Indiae Orientalis, pp:3-4. Parbury, Allen \& Co, London

Yang MM, A Raman (2007). Diversity, richness, and patterns of radiation among gall-inducing psyllids (Hemiptera: Psylloidea) in the Orient and Eastern Palearctic. Orient Ins 41:55-65

Yang MM, LH Liao, MF Lou, WC Chen, SS Huang, GS Tung, YC Weng, CC Shen (2006). Diversity, biology, and nutritional adaptation of psyllids and their galls in Taiwan. In: Galling Arthropods and their Associates, pp:33-42. Ozaki K, J Yukawa, T Ohgushi, PW Price, (Eds.). Springers, Japan

Zendedel A, D Bucrkhardt, L Fekrat, S Manzar, HS Namaghi (2016). An Annotated checklist of the jumping plant-lice (Hemiptera: Psylloidea) of Iran. J Entomol Res Soc 18:37-55 